\title{
Bio-guided fractionation of extracts of Geranium robertianum L.: Relationship between phenolic profile and biological activity
}

\author{
V.C. Graça $a^{\mathrm{a}, \mathrm{b}, \mathrm{c}}$, Lillian Barros ${ }^{\mathrm{b}}$, Ricardo C. Calhelha ${ }^{\mathrm{b}}$, Maria Inês Dias ${ }^{\mathrm{b}}$, Isabel C.F.R. Ferreira ${ }^{\mathrm{b}, *}$, \\ P.F. Santos ${ }^{\mathrm{c}, *}$

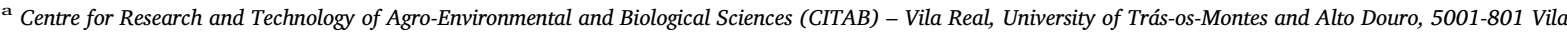 \\ Real, Portugal \\ b Centro de Investigação de Montanha (CIMO), ESA, Instituto Politécnico de Bragança, Campus de Santa Apolónia, $5300-253$ Bragança, Portugal \\ c Chemistry Center - Vila Real (CQVR), University of Trás-os-Montes and Alto Douro, 5001-801 Vila Real, Portugal
}

\section{A R T I C L E I N F O}

\section{Keywords:}

Geranium robertianum $\mathrm{L}$

Fractionation

Antioxidant activity

Antiproliferative activity

Phenolic compounds

\begin{abstract}
A B S T R A C T
Geranium robertianum L. is used in folk medicine and herbalism practice for the treatment of a variety of ailments. Recently, we studied the bioactivity of several aqueous and organic extracts of this plant. In this work, the more active extracts were fractionated and the fractions evaluated for their antioxidant activity and cytotoxicity against several human tumor cell lines and non-tumor porcine liver primary cells. Some of the fractions from the acetone extract consistently displayed low $\mathrm{EC}_{50}$ and $\mathrm{GI}_{50}$ values and presented the higher contents of total phenolic compounds in comparison to other fractions. The phenolic compounds profile of the fractions was determined. The bio-guided fractionation of the extracts resulted in several fractions with improved bioactivity relative to the corresponding extracts. Their lower compositional complexity allowed the identification of more than two dozen compounds, to the best of our knowledge, so far not reported in G. robertianum.
\end{abstract}

\section{Introduction}

Geranium robertianum L., commonly known as Herb Robert or Red Robin, belongs to Geraniaceae family and is commonly found in woodlands, waste lands, roadsides, hedge banks or old walls (Cunha et al., 2012). Widely spread across Europe, it can also found in Asia, Africa and America (Allen and Hatfield, 2004). This plant has been used in folk medicine and herbalism practice for the treatment of a variety of digestive system disorders, and also as anti-inflammatory, haemostatic, antidiabetic, antibacterial, antiallergic, anti-cancer and diuretic (Graça et al., 2016a).

As a consequence of the lifestyle, the contemporary society is subjected to continuous exposure to various sources of aggressors which can provoke an increase in the production of reactive oxygen species (ROS). Free radicals have been implicated in the onset of various diseases such as cardiovascular diseases, cancer, cataracts, age-related decline in the immune system, and degenerative diseases. Antioxidants have the ability to neutralize these species, protecting cells (Aprioku, 2013; Carocho and Ferreira, 2013; Rubió et al., 2013).

G. robertianum contains many biologically active compounds, mostly polyphenols (Amaral et al., 2009; Fodorea et al., 2005; Ivancheva and Petrova, 2000; Kartnig and Bucar-Stachel, 1991;

\footnotetext{
* Corresponding authors.

E-mail addresses: iferreira@ipb.pt (I.C.F.R. Ferreira), psantos@utad.pt (P.F. Santos).
}

Kobakhidze and Alaniya, 2004; Neagu et al., 2013; Paun et al., 2011, 2012, 2014).

The antioxidant effect of phenolic compounds is mainly due to their redox properties and their capacity to block the production of ROS such as superoxide, hydrogen peroxide and hydroxyl, preventing, for example, DNA oxidative damage and/or lipid peroxidation (Rubió et al., 2013). Flavonoids are the main phenolic compounds found in G. robertianum (Graça et al., 2016a), and their effects on human health are very often ascribed to their potential ability to act by diminishing free radical steady-state concentration in biological systems and so providing antioxidant protection (Galleano et al., 2010).

The antioxidant activity and the cytotoxicity against several human tumor cell lines and non-tumor porcine liver primary cells of several aqueous and organic extracts of Geranium robertianum L. were recently evaluated by our research group (Graça et al., 2016b). In the present work, the more active extracts - ethyl acetate, acetone and methanol - were fractionated by gradient elution column chromatography on silica gel and the resulting fractions were evaluated for their bioactive properties, namely the antioxidant and antiproliferative properties. The profile of phenolic compounds of the different fractions was determined and correlated with their bioactivity. 


\section{Materials and methods}

\subsection{Sample}

Geranium robertianum L. was collected in França, Serra de Montesinho, Bragança, Northeastern Portugal, in May 2015. The botanical identification was confirmed by Ana Maria Carvalho from the Department of Biology and Biotechnology of the School of Agriculture, Polytechnic Institute of Bragança (Trás-os-Montes, Portugal). Voucher specimens (ETBO 62 May 2015) are deposited at the herbarium of the Escola Superior Agrária de Bragança (BRESA). The ethyl acetate, acetone and methanol extracts were obtained by sequential extraction of the whole plant, as described previously (Graça et al., 2016b).

\subsection{Standards and reagents}

Silica gel $0.060-0.200 \mathrm{~mm}, 60$ A was obtained from Acros Organics (Geel, Belgium). Acetonitrile was of HPLC grade from Fisher Scientific (Lisbon, Portugal). Trolox (6-hydroxy-2,5,7,8-tetramethylchroman-2carboxylic acid), acetic acid, formic acid, ellipticine, sulphorhodamine B (SRB), trypan blue, trichloroacetic acid (TCA) and Tris was purchased from Sigma-Aldrich (St. Louis, MO, USA). 2,2-Diphenyl-1-picrylhydrazyl (DPPH) was obtained from Alfa Aesar (Ward Hill, MA, USA). Phenolic compound standards were purchased from Extrasynthèse (Genay, France). Foetal bovine serum (FBS), L-glutamine, Hank's balanced salt solution (HBSS), trypsin-EDTA (ethylenediaminetetraacetic acid), penicillin/streptomycin solution $(100 \mathrm{U} / \mathrm{mL}$ and $100 \mathrm{mg} / \mathrm{mL}$, respectively), RPMI-1640 and DMEM media were from Hyclone (Logan, UT, USA). Water was treated in a Milli-Q water purification system (TGI Pure Water Systems, Greenville, SC, USA).

\subsection{Fractionation of extracts}

\subsubsection{Ethyl acetate extract}

The extract $(0.7 \mathrm{~g})$ was dissolved in a minimum of $\mathrm{CH}_{2} \mathrm{Cl}_{2}$, mixed with silica gel, and the mixture evaporated to dryness and placed on the top of silica gel column. The dry-loaded extract was fractionated by gradient elution column chromatography using: $\mathrm{CH}_{2} \mathrm{Cl}_{2} ; \mathrm{CH}_{2} \mathrm{Cl}_{2} / \mathrm{EtOAc}$ - (90:10), (80:20), (70:30), (60:40), (50:50), (40:60), (30:70), (20:80), (10:90); EtOAc, EtOAc/acetone - (90:10), (80:20), (70:30), (60:40), (50:50), (60:40), (70:30), (80:20), (90:10); acetone; acetone/MeOH (90:10), (80:20), (70:30), (60:40), (50:50), (40:60), (30:70), (20:80), (10:90); $\mathrm{MeOH} ; \mathrm{MeOH} /$ formic acid (99:1), (97:3), (95:5). Three hundred and eighty two samples $(\sim 23 \mathrm{~mL}$ each) were collected and grouped in eleven fractions (FEA1-11) according to the similarity of their TLC profiles. The solvent of these final fractions was removed under reduced pressure at $40{ }^{\circ} \mathrm{C}$.

\subsubsection{Acetone extract}

The extract ( 3 gr) was dissolved in acetone, mixed with silica gel, and the mixture evaporated to dryness and placed on the top of a silica gel column. The dry-loaded extract was fractionated by gradient elution column chromatography using: $\mathrm{CH}_{3} \mathrm{Cl} ; \mathrm{CH}_{3} \mathrm{Cl}$ /acetone - (90:10), (80:20), (70:30), (60:40), (50:50), (40:60), (30:70), (20:80), (10:90); acetone; acetone/MeOH - (90:10), (80:20), (70:30), (60:40), (50:50), (40:60), (30:7orated to dryness and placed on the top of a silica gel column. The dry-loaded extract was fractionated by gradient elution column chromatography using: $\mathrm{CH}_{3} \mathrm{Cl} ; \mathrm{CH}_{3} \mathrm{Cl}$ /acetone - (90:10), (80:20), (70:30), (60:40), (50:50), (40:60), (30:70), (20:80), (10:90); acetone; acetone/MeOH - (90:10), (80:20), (70:30), (60:40), (50:50), (40:60), (30:7orated to dryness and placed on the top of a silica gel column. The dry-loaded extract was fractionated by gradient elution column chromatography using: $\mathrm{CH}_{3} \mathrm{Cl} ; \mathrm{CH}_{3} \mathrm{Cl}$ /acetone - (90:10), (80:20), (70:30), (60:40), (50:50), (40:60), (30:70), (20:80), (10:90); acetone; acetone $\mathrm{MeOH}-(90: 10),(80: 20),(70: 30),(60: 40),(50: 50)$, (40:60), (30:70), (20:80), (10:90); $\mathrm{MeOH} ; \mathrm{MeOH} /$ formic acid (99:1),
(97:3), (95:5). Six hundred and seventy-three fractions ( $\sim 23 \mathrm{~mL}$ each) were collected and combined on the basis of their TLC profiles to yield eleven final fractions (FA1-11) which were evaporated to dryness under reduced pressure at $40{ }^{\circ} \mathrm{C}$.

\subsubsection{Methanol extract}

The extract (4 gr) was dissolved in $\mathrm{MeOH}$, mixed with silica gel, and the mixture evaporated to dryness and placed on the top of a silica gel column. The dry-loaded extract was fractionated by gradient elution column chromatography using: $\mathrm{CH}_{2} \mathrm{Cl}_{2} ; \mathrm{CH}_{2} \mathrm{Cl}_{2}$ /EtOAc - (80:20), (40:60), (20:80); EtOAc, EtOAc/acetone - (90:10), (80:20), (70:30), (60:40), (50:50), (40:60), (20:80), (10:90); acetone; acetone/MeOH (90:10), (80:20), (70:30), (60:40), (50:50), (40:60), (30:70), (20:80), (10:90); $\mathrm{MeOH} ; \mathrm{MeOH} /$ formic acid (99:1), (97:3), (95:5). Three hundred and forty three fractions ( $\sim 23 \mathrm{~mL}$ each) were obtained, checked by TLC and those with similar band patterns were combined, yielding thirteen final fractions (FM1-13). The solvent was removed at $40{ }^{\circ} \mathrm{C}$ under reduced pressure.

\subsection{Phenolic profile of the fractions}

The fractions were re-dissolved in water/methanol 80:20 (v/v) (final concentration $5 \mathrm{mg} / \mathrm{mL}$ ). Phenolic compounds were determined by high performance liquid chromatography with a diode array detector, coupled to mass spectrometry using the electrospray ionization interface (HPLC-DAD-ESI/MS), as previously described (Dias et al., 2013; Roriz et al., 2014). Double online detection was carried out with a DAD using $280 \mathrm{~nm}$ and $370 \mathrm{~nm}$ as preferred wavelengths and with a mass spectrometer (MS) connected to the HPLC system via the DAD cell outlet. The phenolic compounds were identified by comparing their retention time, UV-vis and mass spectra with those obtained from standard compounds, when available. Otherwise, peaks were tentatively identified comparing the obtained information with the available data reported in the literature. For quantitative analysis, a calibration curve for each available phenolic standard $(2-100 \mu \mathrm{g} / \mathrm{mL})$ was constructed based on the UV signal. The available phenolic standards are: chlorogenic acid $\left(y=-161172+168823 x, r^{2}=0.9999\right)$; protocatechuic acid $\left(y=27102+214168 x, r^{2}=0.9999\right)$; ferulic acid $\left(y=-185462+633126 x, \quad r^{2}=0.999\right) ; \quad p$-hydroxibenzoic acid $\left(y=173056+208604 x, r^{2}=0.9995\right) ;$ vannilic acid $(y=-28661+$ $\left.29751 x, \quad r^{2}=0.9999\right) ; \quad$ caffeic acid $(y=406369+388345 x$, $\left.r^{2}=0.9939\right) ; \quad$ quercetin-3-O-glucoside $\quad(y=-160173+34843 x$, $\left.r^{2}=0.9998\right) ;$ ellagic acid $\left(y=-317255+26719 x, r^{2}=0.9986\right) ; p$ coumaric acid $\left(y=6966.7+301950 x, r^{2}=0.9999\right)$; quercetin-3-Orutinoside $\left(y=76751+13343 x, r^{2}=0.9998\right)$; kaempferol-3-O-rutinoside $\left(y=30861+11117 x, \quad r^{2}=0.9999\right) ; \quad$ naringenin $(y=$ $\left.78903+18433 x, r^{2}=0.9998\right)$. For the identified phenolic compounds for which a commercial standard was not available, the quantification was performed through the calibration curve of other compound from the same phenolic group. The results are expressed in mg per $g$ of extract.

\subsection{Evaluation of bioactivity of the fractions}

\subsubsection{Antioxidant activity}

Three different in vitro assays were performed using solutions prepared by serial dilution of the stock solutions: scavenging of DPPH $(2,2-$ diphenyl-1-picrylhydrazyl) radicals, reducing power (measured by ferricyanide Prussian blue assay) and inhibition of $\beta$-carotene bleaching as previously described (Dias et al., 2013; Roriz et al., 2014)

\subsubsection{Cytotoxicity in human tumor cell lines and hepatotoxicity in non-} tumor cells

Four human tumor cell lines were tested using solutions prepared by serial dilution of the stock solutions: MCF-7 (breast adenocarcinoma), NCI-H460 (non-small cell lung cancer), HeLa (cervical carcinoma) and 
HepG2 (hepatocellular carcinoma), as previously described (Dias et al., 2013). The hepatotoxicity was evaluated against a non-tumor porcine liver primary cells (PLP2), as described earlier (Barros et al., 2013; Dias et al., 2013).

\subsection{Statistical analysis}

For all the experiments, three samples were analysed and all the assays were carried out in triplicate. The results are expressed as mean values \pm standard deviation (SD). All statistical tests were performed at a $5 \%$ significance level using IBM SPSS Statistics for Windows, version 23.0. (IBM Corp., Armonk, NY, USA). The fulfilment of the oneway ANOVA requirements, specifically the normal distribution of the residuals (data not shown) and the homogeneity of variance, was tested by means of the Shapiro Wilk's and the Levene's tests, respectively. All dependent variables were compared using Tukey's honestly significant difference (HSD) or Tamhane's T2 multiple comparison tests, when homoscedasticity was verified or not, respectively. Furthermore, a Pearson's correlation analysis between the antioxidant activity and all the analysed compounds was carried out, with a $95 \%$ confidence level.

\section{Results and discussion}

\subsection{Analysis of phenolic profile of the fractions}

Peak characteristics and tentative identities are presented in Table 1 and the quantification of each individual compound present in each fraction is presented in Tables 2-4.

The most abundant compounds were assigned to hydrolyzable tannins, being compounds 2, 9, 12, 17, 21, 23, 27 and 35 identified as gallotannins composed by two, three, four or five galloyl moieties linked to glucose. The mass spectra characteristics of these compounds consisted of the deprotonated molecule $\left([\mathrm{M}-\mathrm{H}]^{-}\right.$ions at $m / z 483$ (compound 2), 635 (compounds 9, 12 and 17), 787 (compounds 21, 23 and 27), and 939 (compound 35), with the loss of one or more galloyl groups $(152 \mathrm{u})$ and/or gallic acid (170 u). Compounds 14, 15, 18 ([M $-\mathrm{H}]^{-}$ion at $\left.m / z 951\right)$ and $30\left([\mathrm{M}-\mathrm{H}]^{-}\right.$ion at $m / z$ 933), which were abundantly present in the $G$. robertianum acetone and $\mathrm{MeOH}$ extracts, were tentatively identified as ellagitannins: three isomers of geraniin and castalagin/vescalagin (both with the same molecular weight), respectively. The different chromatographic retention times of compounds 14,15 and 18, presenting the same molecular mass, strongly suggests that they correspond to distinct isomers. These compounds have also been reported in different Geranium species (Okuda et al., 2000; Tuominen, 2013; Tuominen et al., 2013) and previously reported by us in G. robertianum (Graça et al., 2016b). Compound $8\left([\mathrm{M}-\mathrm{H}]^{-}\right.$at $m / z 633$ ) was identified as galloyl-HHDP-glucose, while peaks 19 and $26\left([\mathrm{M}-\mathrm{H}]^{-}\right.$at $\left.m / z 785\right)$ were coherent with two digalloyl-HHDPglucose isomers. The pseudomolecular ion $[\mathrm{M}-\mathrm{H}]^{-}$of compound 32 $(\mathrm{m} / \mathrm{z}$ at 935$)$ might point to a galloyl-bis-HHDP-glucose, although a different fragmentation pattern was described (Dias et al., 2015; Hanhineva et al., 2008). However, the presence of two HHDP moieties in its structure was supported by the loss of an HHDP fragment (302 Da, from the transition $935>633$ ), together with the observation of a product ion at $m / z$ 301. All of these mentioned compounds have already been described in the previously study G. robertianum (Graça et al., 2016b).

Peaks 6 and 38 were tentatively associated to ellagitannins, based on their UV spectra and on the observation of an $\mathrm{MS}^{2}$ fragment ion at $\mathrm{m} / \mathrm{z} 301$ ([HHDP-H $^{-}$). Nevertheless, a full identification of these compounds was not possible being identified as unknown ellagitannins.

Seventeen flavonoid glycosides derivatives were detected nine of which were kaempferol derivatives, seven quercetin derivatives and a narigenin derivative. Peaks 25 (quercetin-3-O-rutinoside), 34 (kaempferol-3-O-rutinoside), 36 (quercetin-3-O-glucoside) and 39 (narigenin7-O-glucoside), were positively identified according to their retention time and UV and mass spectra, in comparison with commercial standards.

Compounds 28, 29, 33, 43, 47, 48, 49 and 50 were identified as kaempferol glycoside derivatives owing to the product ion observed at $m / z$ 285 and UV spectra $\left(\lambda_{\max }\right.$ around $\left.348 \mathrm{~nm}\right)$. Compound $28\left([\mathrm{M}-\mathrm{H}]^{-}\right.$at $m / z$ 765) presented two $\mathrm{MS}^{2}$ fragments, revealing the alternative loss of acetylglucuronyl $(\mathrm{m} / \mathrm{z}$ at $547 ;-42-176 \mathrm{u})$ and malonylglucuronyl $(\mathrm{m} / \mathrm{z}$ at 285 ; -86-176 u) residues, indicating location of each residue on different positions of the aglycone. For compound $29\left([\mathrm{M}-\mathrm{H}]^{-}\right.$at $m / z$ 739) only one $\mathrm{MS}^{2}$ fragment was present $(m / z$ at $285 ;-146-146-162 u)$ suggesting that the three sugars were linked together. No information about the identity of the sugar moieties and location onto the aglycone could be obtained, so, these compounds were tentatively identified as kaempferol-O-acetylglucuronyl-Omalonylglucuronide and kaempferol-O-deoxyhexosyl-(deoxyhexosyl-hexoside), respectively. Mass characteristics of peak $47\left([\mathrm{M}-\mathrm{H}]^{-}\right.$at $\left.\mathrm{m} / \mathrm{z} 609\right), 43$ and $49\left([\mathrm{M}-\mathrm{H}]^{-}\right.$at $\left.m / z 637\right)$ indicated that these compounds corresponds to kaempferol derivatives bearing two hexosyl (-162-162 u) and glucuronyl $(-176-176 \mathrm{u})$ residues, respectively. The observation of only one $\mathrm{MS}^{2}$ fragment at $m / z 285$, also indicated that the residue moieties were linked together. Thus, these compounds were tentatively identified as kaempferol-Odihexoside and kaempferol-O-diglucuronide. Similarly, compounds 48 ([M$\mathrm{H}]^{-}$at $\left.m / z 607\right)$ and $50\left([\mathrm{M}-\mathrm{H}]^{-}\right.$at $\left.m / z 591\right)$, also released one fragment at $\mathrm{m} / z 285$, from the respective losses of deoxyhexosyl-glucuronide ( $322 \mathrm{u}$ ) and acetylpentosyl-pentoside (306 u) moieties, thus being tentatively assigned as kaempferol-O-deoxyhexosyl-glucuronide and kaempferol-O-acetylpentosylpentoside. Compound 33 presented the same molecular weight as compound 34 (kaempferol-3-O-rutinoside), but with an earlier retention time, thus being assigned to kaempferol-O-deoxyhexosyl-hexoside.

Compounds 20, 24, 31, 37 and 41 were identified as quercetin glycosides derivatives based on their UV spectra $\left(\lambda_{\max }\right.$ around $\left.350 \mathrm{~nm}\right)$ and the production of a $\mathrm{MS}^{2}$ fragment ion at $m / z 301$. Tentative identities of these compounds were assigned based on their pseudomolecular ions using a similar reasoning as for kaempferol derivatives. Thus, compound $20\left([\mathrm{M}-\mathrm{H}]^{-}\right.$at $\left.m / z 755\right), 24$ and 31 ([M-H] ${ }^{-}$at $\left.m / z 609\right)$ could correspond to quercetin- $O$-dideoxyhexosyl-hexoside and quercetin-O-deoxyhexosyl-hexoside, respectively, whereas peaks 37 ([M$\mathrm{H}]^{-}$at $\left.m / z 597\right)$ and $41\left([\mathrm{M}-\mathrm{H}]^{-}\right.$at $\left.m / z 623\right)$ should correspond to quercetin-O-deoxyhexosyl-pentoside and quercetin- $O$-deoxyhexosylglucuronide, respectively.

Peaks 20, 25, 29, 33, 34 and 36, correspond to different quercetin and kaempferol glycosides that have been previously described in $G$. robertianum (Fodorea et al., 2005; Graça et al., 2016b; Ivancheva and Petrova, 2000; Kartnig and Bucar-Stachel, 1991; Kobakhidze and Alaniya, 2004).

Twelve phenolic acids were detected, five hydroxybenzoic acids (compounds 3, 11, 13, 40 and 42) and seven hydroxycinnamic acid and derivatives (compounds 1, 4, 5, 7, 10, 16 and 22). Protocatechuic acid (compound 3), 5-O-caffeoylquinic acid (compound 5), p-hydroxybenzoic acid (compound 11), vanillic acid (compound 13), p-coumaric acid (compound 22) were positively identified according to their retention time and UV and mass spectra, in comparison with commercial standards. 3-O-Caffeoylquinic acid (compound 1), 3-p-coumaroylquinic acid (compound 4), 3-O- and 4-O-feruloylquinic acids (compounds 7 and 10, respectively), were tentatively identified taking into account their fragmentation pattern and relative intensities similar to those reported by Clifford et al. (2003) and Clifford et al. (2005). Compound $16\left([\mathrm{M}-\mathrm{H}]^{-}\right.$at $\left.m / z 335\right)$ was tentatively identified as a caffeoylshikimic acid due to its similar fragmentation pattern described by Al-Ayed (2015) and Bastos et al. (2007). Compounds $40\left([\mathrm{M}-\mathrm{H}]^{-}\right.$at $m / z 625)$ and $42\left([\mathrm{M}-\mathrm{H}]^{-}\right.$at $\left.m / z 591\right)$, both produced a major $\mathrm{MS}^{2}$ fragment ion at $m / z 301$, which can be interpreted as corresponding to deprotonated ellagic acid; in the case of compound 40, it lost two hexosyl moieties $(-324 \mathrm{u})$, which was tentatively identified as an ellagic acid dihexoside. No full identity was obtained for compound 42, being assigned as ellagic acid derivative. Moreover, no tentative identification was possible to achieve for compounds 44,45 and 46, remaining as 
Table 1

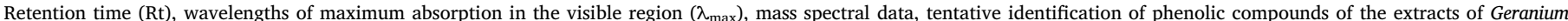
robertianum $\mathrm{L}$.

\begin{tabular}{|c|c|c|c|c|c|c|c|c|}
\hline Peak & Rt (min) & $\lambda_{\max }(\mathrm{nm})$ & $\begin{array}{l}\text { Molecular ion [M- } \\
\mathrm{H}]^{-}(m / z)\end{array}$ & $\operatorname{MS}^{2}(m / z)$ & Tentative identification & $\begin{array}{l}\text { Ethyl } \\
\text { Acetate }\end{array}$ & Acetone & Metanol \\
\hline 1 & 4.88 & 328 & 353 & 191(64),179(32),161(3),135(40) & 3-O-Caffeoylquinic acid & d & $\mathrm{d}$ & $\mathrm{d}$ \\
\hline 2 & 4.57 & 276 & 483 & $331(13), 313(23), 271(11), 169(6)$ & Digalloyl-glucose & nd & $\mathrm{d}$ & nd \\
\hline 3 & 5.76 & $260, \operatorname{sh} 294$ & 153 & $109(100)$ & Protocatechuic acid ${ }^{\mathrm{a}}$ & $\mathrm{d}$ & nd & $\mathrm{d}$ \\
\hline 4 & 6.52 & 312 & 337 & $191(4), 173(5), 163(100), 119(50)$ & 3-p-Coumaroylquinic acid ${ }^{\mathrm{a}}$ & nd & $\mathrm{d}$ & $\mathrm{d}$ \\
\hline 5 & 7.01 & 320 & 353 & $191(100), 179(5), 173(6), 161(5), 135(5)$ & 5-O-Caffeoylquinic acid & $\mathrm{d}$ & nd & $\mathrm{d}$ \\
\hline 6 & 7.37 & $224 / \operatorname{sh} 286$ & 815 & $797(25), 753(8), 725(6), 301(60)$ & Unknown ellagitannin & nd & $\mathrm{d}$ & nd \\
\hline 7 & 7.56 & 323 & 367 & $193(100), 191(3), 173(3), 148(6), 134(4), 107(50)$ & 3-O-Feruloylquinic acid ${ }^{\mathrm{a}}$ & $\mathrm{d}$ & $\mathrm{d}$ & $\mathrm{d}$ \\
\hline 8 & 8.01 & 274 & 633 & $463(34), 301(100)$ & Galloyl-HHDP-glucose & nd & $\mathrm{d}$ & $\mathrm{d}$ \\
\hline 9 & 8.16 & 278 & 635 & $483(6), 465(100), 313(26), 295(5), 169(5)$ & Trigalloyl-glucose & nd & $\mathrm{d}$ & nd \\
\hline 10 & 8.66 & 328 & 367 & 193(22),191(21),173(3),161(100),135(13) & 4-O-Feruloylquinic acid ${ }^{\mathrm{a}}$ & nd & nd & $\mathrm{d}$ \\
\hline 11 & 8.69 & 256 & 137 & $93(100)$ & $p$-Hydroxybenzoic acid ${ }^{\mathrm{a}}$ & $\mathrm{d}$ & nd & nd \\
\hline 12 & 9.63 & 278 & 635 & $483(6), 465(100), 313(26), 295(5), 169(5)$ & Trigalloyl-glucose & nd & nd & $\mathrm{d}$ \\
\hline 13 & 10.35 & $261, \operatorname{sh} 292$ & 167 & $108(100)$ & Vanillic acid $^{\mathrm{a}}$ & $\mathrm{d}$ & nd & nd \\
\hline 14 & 11.42 & 270 & 951 & $933(70), 633(3), 481(3), 451(4), 301(39)$ & Geraniin isomer 1 & nd & $\mathrm{d}$ & $\mathrm{d}$ \\
\hline 15 & 12.53 & 270 & 951 & $933(70), 633(3), 481(3), 451(4), 301(39)$ & Geraniin isomer $2^{\mathrm{a}}$ & nd & $\mathrm{d}$ & nd \\
\hline 16 & 12.76 & 326 & 335 & $179(100), 135(16)$ & Caffeoylshikimic acid & $\mathrm{d}$ & nd & nd \\
\hline 17 & 12.81 & 284 & 635 & $483(11), 465(35), 343(17), 295(5), 169(20)$ & Trigalloyl-glucose & nd & $\mathrm{d}$ & nd \\
\hline 18 & 13.12 & 270 & 951 & $933(70), 633(3), 481(3), 451(4), 301(39)$ & Geraniin isomer $3^{\mathrm{a}}$ & nd & $\mathrm{d}$ & nd \\
\hline 19 & 14.31 & 280 & 785 & $483(12), 301(100)$ & Digalloyl-HHDP-glucose & nd & $\mathrm{d}$ & nd \\
\hline 20 & 14.83 & 356 & 755 & $301(100)$ & Quercetin-O-dideoxyhexosyl-hexoside & $\mathrm{d}$ & $\mathrm{d}$ & $\mathrm{d}$ \\
\hline 21 & 14.88 & 280 & 787 & $635(22), 617(12), 465(4), 169(13)$ & Tetragalloyl-glucose & $\mathrm{d}$ & $\mathrm{d}$ & $\mathrm{d}$ \\
\hline 22 & 15.18 & 310 & 163 & $119(100)$ & $p$-Coumaric acid & $\mathrm{d}$ & nd & $\mathrm{d}$ \\
\hline 23 & 15.77 & 280 & 787 & $635(22), 617(12), 465(4), 169(13)$ & Tetragalloyl-glucose $\mathrm{a}^{\mathrm{a}}$ & nd & $\mathrm{d}$ & nd \\
\hline 24 & 15.97 & 350 & 609 & $301(100)$ & Quercetin-O-deoxyhexosyl-hexoside ${ }^{\text {a }}$ & nd & $\mathrm{d}$ & $\mathrm{d}$ \\
\hline 25 & 16.37 & 354 & 609 & $301(100)$ & Quercetin-3-O-rutinoside & $\mathrm{d}$ & $\mathrm{d}$ & $\mathrm{d}$ \\
\hline 26 & 16.39 & 278 & 785 & $633(5), 615(5), 483(15), 301(84), 275(6)$ & Digalloyl-HHDP-glucose & nd & $\mathrm{d}$ & nd \\
\hline 27 & 16.82 & 280 & 787 & $635(5), 617(100), 465(7), 169(5)$ & Tetragalloyl-glucose & nd & $\mathrm{d}$ & $\mathrm{d}$ \\
\hline 28 & 16.95 & 348 & 765 & $547(26), 285(100)$ & $\begin{array}{l}\text { Kaempferol-O-acetylglucuronyl-O- } \\
\text { malonylglucuronide }^{\mathrm{a}}\end{array}$ & $\mathrm{d}$ & nd & nd \\
\hline 29 & 17.19 & 350 & 739 & $285(100)$ & $\begin{array}{l}\text { Kaempferol-O-dideoxyhexosyl- } \\
\text { hexoside }\end{array}$ & $\mathrm{d}$ & $\mathrm{d}$ & $\mathrm{d}$ \\
\hline 30 & 18.08 & $226 / \operatorname{sh} 280$ & 933 & $915(12), 765(10), 631(3), 613(3), 463(10), 301(42)$ & Castalagin/Vescalagin & nd & $\mathrm{d}$ & nd \\
\hline 31 & 18.15 & 353 & 609 & $301(100)$ & 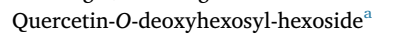 & $\mathrm{d}$ & $\mathrm{d}$ & $\mathrm{d}$ \\
\hline 32 & 18.18 & 277 & 935 & $633(6), 301(21)$ & Galloyl-bis-HHDP-glucose ${ }^{\mathrm{a}}$ & nd & $\mathrm{d}$ & nd \\
\hline 33 & 18.77 & 350 & 593 & $285(100)$ & Kaempferol-O-deoxyhexosyl-hexoside & $\mathrm{d}$ & $\mathrm{d}$ & $\mathrm{d}$ \\
\hline 34 & 18.82 & 350 & 593 & $285(100)$ & Kaempferol-3-O-rutinoside & $\mathrm{d}$ & $\mathrm{d}$ & nd \\
\hline 35 & 19.3 & $269 / \operatorname{sh} 365$ & 939 & $787(3), 769(76), 617(9), 465(3)$ & Pentagalloyl glucose $\mathrm{e}^{\mathrm{a}}$ & nd & $\mathrm{d}$ & nd \\
\hline 36 & 19.32 & 358 & 463 & $301(100)$ & Quercetin-3-O-glucoside & nd & nd & $\mathrm{d}$ \\
\hline 37 & 19.63 & 347 & 579 & $301(100)$ & Quercetin-O-deoxyhexosyl-pentoside ${ }^{\text {a }}$ & nd & nd & $\mathrm{d}$ \\
\hline 38 & 20.64 & 280 & 935 & $917(16), 767(21), 749(11), 465(8), 301(23)$ & Unknown ellagitannin & nd & $\mathrm{d}$ & nd \\
\hline 39 & 21.3 & 283 & 433 & $271(100)$ & Naringenin $-7-O$-glucoside ${ }^{\text {a }}$ & $\mathrm{d}$ & nd & nd \\
\hline 40 & 21.85 & 370 & 625 & $301(100)$ & Ellagic acid dihexoside $^{a}$ & nd & nd & $\mathrm{d}$ \\
\hline 41 & 23.09 & 355 & 623 & $301(100)$ & $\begin{array}{l}\text { Quercetin-O-deoxyhexosyl- } \\
\text { glucuronide }^{\text {a }}\end{array}$ & $\mathrm{d}$ & nd & $\mathrm{d}$ \\
\hline 42 & 23.26 & 365 & 591 & $301(100), 179(50), 151(30)$ & Ellagic acid derivative & nd & $\mathrm{d}$ & nd \\
\hline 43 & 24.34 & 340 & 637 & $285(100)$ & Kaempferol-O-diglucuronide $^{a}$ & nd & nd & $\mathrm{d}$ \\
\hline 44 & 24.69 & 312 & 433 & $307(100)$ & Unknown & $\mathrm{d}$ & nd & $\mathrm{d}$ \\
\hline 45 & 24.87 & $259 / \operatorname{sh} 305$ & 579 & $459(13), 441(73), 289(100), 169(86)$ & Unknown & nd & nd & $\mathrm{d}$ \\
\hline 46 & 25.42 & 307 & 433 & $307(100)$ & Unknown & $\mathrm{d}$ & nd & nd \\
\hline 47 & 25.85 & 351 & 609 & $285(100)$ & Kaempferol-O-dihexoside $\mathrm{a}^{\mathrm{a}}$ & nd & nd & $\mathrm{d}$ \\
\hline 48 & 27.25 & 351 & 607 & $285(100)$ & $\begin{array}{l}\text { Kaempferol-O-deoxyhexosyl- } \\
\text { glucuronide }^{\mathrm{a}}\end{array}$ & $\mathrm{d}$ & nd & $\mathrm{d}$ \\
\hline 49 & 30.94 & 349 & 637 & $285(100)$ & Kaempferol-O-diglucuronide ${ }^{a}$ & nd & nd & $\mathrm{d}$ \\
\hline 50 & 31.48 & 348 & 591 & $285(100)$ & $\begin{array}{l}\text { Kaempferol-O-acetylpentosyl- } \\
\text { pentoside }^{\mathrm{a}}\end{array}$ & nd & nd & $\mathrm{d}$ \\
\hline
\end{tabular}

d - detected. nd - not detected.

${ }^{\text {a }}$ Hitherto unreported in $G$. robertianum.

unknown compounds. 5-O-Caffeoylquinic acid, 3-O-caffeoylquinic acid, p-coumaric acid have been reported in some Geranium species Paun et al., 2011, 2012; Tuominen, 2013; Tuominen et al., 2013).

In this work, the lower compositional complexity of the fractions obtained compared with the whole extracts turned possible the identification of more than two dozens of compounds, mostly quercetin and kampferol glycosides, phenolic acids and galloyl tannins (Table 1), which, to the best of our knowlege, have not hitherto been reported in G. robertianum.

\subsection{Evaluation of bioactivity of the fractions}

The antioxidant properties of the fractions obtained from the most bioactive extracts of $G$. robertianum (EtOAc, acetone and $\mathrm{MeOH}$ ) were evaluated by three different assays: DPPH radical scavenging capacity, reducing power and $\beta$-carotene bleaching inhibition. The results are shown in Table 5. The cytotoxic activities of the same fractions were tested against four human tumor cell lines (i.e., MCF-7, NCI-H460, HeLa and HepG2) and in a non-tumor primary culture (porcine liver primary cell culture). The results are presented in Table 5. 
Table 2

Quantification of phenolic compounds present in the fractions of ethyl acetate extract of Geranium robertianum L. ${ }^{\mathrm{a}}$.

\begin{tabular}{|c|c|c|c|c|c|c|c|}
\hline Peak & Tentative identification & $\begin{array}{l}\text { FEA5 } \\
\mathrm{mg} / \mathrm{g}\end{array}$ & FEA6 & FEA7 & FEA8 & FEA9 & FEA10 \\
\hline 1 & 3-O-Caffeoylquinic acid & - & - & - & - & - & $1.335 \pm 0.006$ \\
\hline 3 & Protocatechuic acid & - & $0.137 \pm 0.002$ & $0.23 \pm 0.01$ & $0.1302 \pm 0.0007$ & - & - \\
\hline 5 & 5-O-Caffeoylquinic acid & - & - & - & $0.259 \pm 0.003$ & - & - \\
\hline 7 & 3-O-Ferulouylquinic acid & - & - & - & $0.2425 \pm 0.0009$ & - & - \\
\hline 11 & $p$-Hydroxybenzoic acid & $2.67 \pm 0.02$ & $0.399 \pm 0.009$ & $0.173 \pm 0.008$ & - & - & - \\
\hline 13 & Vanillic acid & $0.348 \pm 0.007$ & - & - & - & - & - \\
\hline 16 & Caffeoylshikimic acid & - & $\operatorname{tr}$ & - & - & - & - \\
\hline 20 & Quercetin-O-dideoxyhexosyl-hexoside & - & - & - & $0.8924 \pm 0.0001$ & - & - \\
\hline 21 & Tetragalloyl-glucose & - & - & - & - & $2.636 \pm 0.001$ & - \\
\hline 22 & $p$-Coumaric acid & $0.65 \pm 0.01$ & $\operatorname{tr}$ & - & - & - & - \\
\hline 25 & Quercetin-3-O-rutinoside & - & - & - & $\operatorname{tr}$ & $\operatorname{tr}$ & - \\
\hline 28 & Kaempferol-O-acylglucuronyl-O-malonylglucuronide & - & $\operatorname{tr}$ & - & - & - & - \\
\hline 29 & Kaempferol-O-dideoxyhexosyl-hexoside & - & - & - & - & $\operatorname{tr}$ & - \\
\hline 31 & Quercetin-O-deoxyhexosyl-hexoside & - & - & - & $0.8916 \pm 0.0001$ & - & - \\
\hline 33 & Kaempferol-O-deoxyhexosyl-hexoside & - & - & - & & $\operatorname{tr}$ & - \\
\hline 34 & Kaempferol-3-O-rutinoside & - & - & - & - & $\operatorname{tr}$ & - \\
\hline 39 & Naringenin-7-O-glucoside & - & $\operatorname{tr}$ & - & - & - & - \\
\hline 41 & Quercetin-O-deoxyhexosyl-glucuronide & - & - & - & $0.9250 \pm 0.0009$ & $0.9421 \pm 0.0004$ & - \\
\hline 44 & Unknown & - & - & - & - & - & - \\
\hline 46 & Unknown & - & - & - & - & - & - \\
\hline 48 & Kaempferol-O-deoxyhexosyl-glucuronide & - & $2.32 \pm 0.05$ & - & $\operatorname{tr}$ & $\operatorname{tr}$ & $1.28 \pm 0.02$ \\
\hline TPA & & $3.66 \pm 0.04$ & $0.536 \pm 0.007$ & $0.404 \pm 0.004$ & $0.631 \pm 0.003$ & - & $1.335 \pm 0.006$ \\
\hline TED & & - & - & - & - & $2.6365 \pm 0.0008$ & - \\
\hline $\mathrm{TF}$ & & - & $2.32 \pm 0.05$ & - & $2.7090 \pm 0.0009$ & $0.9421 \pm 0.0004$ & $1.28 \pm 0.02$ \\
\hline TPC & & $3.66 \pm 0.04$ & $2.85 \pm 0.04$ & $0.483 \pm 0.002$ & $3.341 \pm 0.004$ & $3.579 \pm 0.001$ & $2.61 \pm 0.03$ \\
\hline
\end{tabular}

TPA - Total phenolic acids. TED - Total ellagitannins derivate. TF - Total flavonoids. TPC - Total phenolic compounds. FEA - fractions of ethyl acetate extract.

${ }^{a}$ Fractions FEA1, FEA2, FEA3 and FEA4 did not present phenolic compounds.

Table 3

Quantification of phenolic compounds present in the fractions of acetone extract of Geranium robertianum L. .

\begin{tabular}{|c|c|c|c|c|c|c|c|c|c|}
\hline Peak & Tentative identification & $\begin{array}{l}\mathrm{FA} 4 \\
\mathrm{mg} / \mathrm{g}\end{array}$ & FA5 & FA6 & FA7 & FA8 & FA9 & FA10 & FA11 \\
\hline 1 & 3-O-Caffeoylquinic acid & - & - & $0.36 \pm 0.01$ & $3.3 \pm 0.05$ & $11.5 \pm 0.5$ & $1.98 \pm 0.05$ & $0.60 \pm 0.01$ & - \\
\hline 2 & Digalloyl-glucose & $2.84 \pm 0.02$ & $4.22 \pm 0.03$ & - & - & - & - & - & - \\
\hline 4 & 3-p-Coumarouylquinic acid & - & - & - & $0.40 \pm 0.01$ & - & - & - & - \\
\hline 6 & Unknown ellagitannin & $4.3 \pm 0.1$ & $2.77 \pm 0.04$ & $2.57 \pm 0.04$ & - & - & - & - & - \\
\hline 7 & 3-O-Ferulouylquinic acid & - & - & - & $0.352 \pm 0.001$ & $1.4 \pm 0.0$ & - & - & - \\
\hline 8 & Galloyl-HHDP-glucose & - & - & - & - & - & - & - & $4.09 \pm 0.03$ \\
\hline 9 & Trigalloyl-glucose & $5.82 \pm 0.06$ & $3.1 \pm 0.1$ & - & - & - & - & - & - \\
\hline 14 & Geraniin isomer 1 & $9.3 \pm 0.2$ & $28.6 \pm 0.4$ & $7.68 \pm 0.07$ & $7.2 \pm 0.1$ & $15.1 \pm 0.6$ & - & - & $7.69 \pm 0.25$ \\
\hline 15 & Geraniin isomer 2 & - & - & $4.94 \pm 0.01$ & $4.89 \pm 0.07$ & - & - & - & - \\
\hline 17 & Trigalloyl-glucose & $3.37 \pm 0.03$ & - & - & - & - & - & - & - \\
\hline 18 & Geraniin isomer 3 & - & $2.68 \pm 0.05$ & $3.51 \pm 0.04$ & $3.4 \pm 0.1$ & - & - & - & - \\
\hline 19 & Digalloyl-HHDP-glucose & $4.44 \pm 0.03$ & $2.68 \pm 0.04$ & - & - & - & - & - & - \\
\hline 20 & Quercetin-O-dideoxyhexosyl-hexoside & - & - & - & - & $2.39 \pm 0.03$ & - & - & - \\
\hline 21 & Tetragalloyl-glucose & $6.13 \pm 0.05$ & $3.15 \pm 0.02$ & - & - & - & - & - & - \\
\hline 23 & Tetragalloyl-glucose & $4.08 \pm 0.07$ & $3.96 \pm 0.04$ & - & - & - & - & - & - \\
\hline 24 & Quercetin-O-deoxyhexosyl-hexoside & - & - & - & $1.16 \pm 0.01$ & $1.085 \pm 0.004$ & - & - & - \\
\hline 25 & Quercetin-3-O-rutinoside & - & - & - & $3.39 \pm 0.06$ & $\operatorname{tr}$ & - & - & - \\
\hline 26 & Digalloyl-HHDP-glucose & $3.81 \pm 0.02$ & - & - & - & - & - & - & - \\
\hline 27 & Tetragalloyl-glucose & $7.6 \pm 0.3$ & $4.10 \pm 0.04$ & - & - & - & - & - & - \\
\hline 29 & $\begin{array}{l}\text { Kaempferol-O-dideoxyhexosyl- } \\
\text { hexoside }\end{array}$ & - & - & - & $0.04 \pm 0.00$ & $1.054 \pm 0.001$ & - & - & - \\
\hline 30 & Castalagin/Vescalagin & $23.6 \pm 0.6$ & $31.5 \pm 0.1$ & $7.0 \pm 0.2$ & - & - & - & - & - \\
\hline 31 & Quercetin-O-deoxyhexosyl-hexoside & - & - & - & $1.96 \pm 0.04$ & - & - & - & - \\
\hline 32 & Galloyl-bis-HHDP-glucose & - & - & - & - & $15.5 \pm 0.3$ & - & - & $7.58 \pm 0.03$ \\
\hline 33 & Kaempferol-O-deoxyhexosyl-hexoside & - & - & - & $2.61 \pm 0.07$ & - & - & - & - \\
\hline 34 & Kaempferol-3-O-rutinoside & - & - & - & $1.63 \pm 0.02$ & - & - & - & - \\
\hline 35 & Pentagalloyl glucose & $3.4 \pm 0.1$ & $7.03 \pm 0.08$ & - & - & - & - & - & - \\
\hline 38 & Unknown ellagitannin & $5.2 \pm 0.1$ & $3.48 \pm 0.02$ & - & - & - & - & - & - \\
\hline 42 & Ellagic acid derivative & $7.3 \pm 0.2$ & - & - & - & - & - & - & - \\
\hline TPA & & - & - & $0.36 \pm 0.01$ & $4.1 \pm 0.1$ & $12.9 \pm 0.5$ & $1.98 \pm 0.05$ & $0.60 \pm 0.01$ & - \\
\hline TED & & $91 \pm 1$ & $97.3 \pm 0.3$ & $25.67 \pm 0.02$ & $15.5 \pm 0.3$ & $30.7 \pm 0.3$ & - & - & $19.35 \pm 0.31$ \\
\hline $\mathrm{TF}$ & & - & - & - & $10.8 \pm 0.2$ & $4.53 \pm 0.03$ & - & - & - \\
\hline TPC & & $91 \pm 1$ & $97.3 \pm 0.3$ & $26.03 \pm 0.03$ & $30.4 \pm 0.5$ & $48.1 \pm 0.9$ & $1.98 \pm 0.05$ & $0.60 \pm 0.01$ & $19.35 \pm 0.3$ \\
\hline
\end{tabular}

TPA - Total phenolic acids. TED - Total ellagitannins derivate. TF - Total flavonoids. TPC - Total phenolic compounds. FA - fractions of acetone extract.

${ }^{\text {a }}$ Fractions FA1, FA2 and FA3 did not present phenolic compounds. 


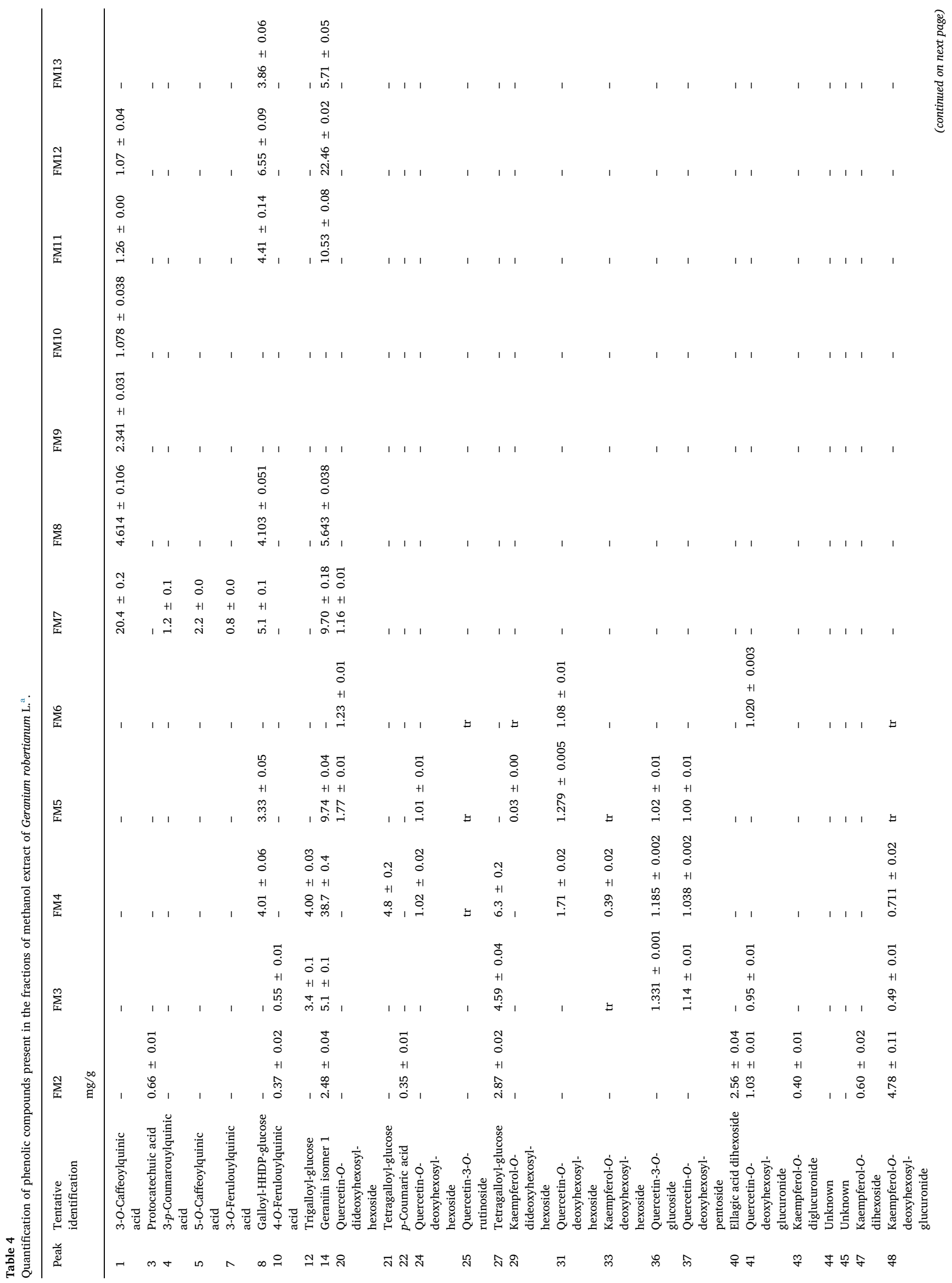


In general all fractions resulting from the acetone extract (FA1FA11) exhibited bioactivity. The most bioactive ones were FA4, FA5 and FA8, which also presented the higher contents of total phenolic compounds (91.0, 97.3 and $48.1 \mathrm{mg} / \mathrm{g}$, respectively, Table 3) when compared to the other fractions. Amongst those, fraction FA4 displayed particularly low hepatotoxicity $\left(\mathrm{IC}_{50}>400 \mu \mathrm{g} / \mathrm{mL}\right.$ ).

Fractions FA4 and FA5 showed to contain only ellagitannins and the higher amount of these group of compounds among all the tested fractions. Compound 30, which was tentatively identified as castalagin or vescalagin, was the main compound of these fractions $(23.6 \mathrm{mg} / \mathrm{g}$ in FA4 and $31.5 \mathrm{mg} / \mathrm{g}$ in FA5, Table 3). The antioxidant and cytotoxic properties of both castalagin and vescalagin have been previously described (Fernandes et al., 2009; Fridrich et al., 2008; Jordão et al., 2012). In each of the fractions FA4, FA5 and FA8 compound 14, an isomer of geraniin, was the second most abundant compound present (9.3, 26.6 and $15.1 \mathrm{mg} / \mathrm{g}$, respectively, Table 3). Geraniin has been described as the main phenolic compound in various Geranium species (Harborne and Williams, 2002; Okuda et al., 1980, 1979, 2000; Piwowarski et al., 2014; Tuominen, 2013; Tuominen et al., 2013; Wu et al., 2011) and is endowed with several beneficial biological activities (Cheng et al., 2017). Apart from the identified ellagitannins, FA8 showed to contain also some phenolic acids and flavonoids. In this fraction, compound 32, tentatively identified as galloyl-bis-HHDP-glucose, was the main compound present $(15.5 \mathrm{mg} / \mathrm{g}$, Table 3$)$.

The $\mathrm{MeOH}$ extract fractionation resulted in thirteen fractions (FM113). In general, all of them possessed biological activity. FM3 and FM4 were the fractions that exhibited, simultaneously, the best antioxidant and cytotoxic activities. FM2 also exhibited good cytotoxic activity but poor/moderate antioxidant activity, except for the DPPH assay. FM4 showed additionally the highest content of total phenolic compounds $(63.9 \mathrm{mg} / \mathrm{g}$, Table 4), but this direct relation activity/content of phenolic compounds was not observed for the remaining more bioactive fractions.

Fraction FM2 possessed approximate amounts of ellagitannins and flavonoids (11.43 and $9.30 \mathrm{mg} / \mathrm{g}$, respectively, Table 4), and also some phenolic acids (2.23 mg/g, Table 4). Compound 48, which was tentatively identified as kaempferol-O-deoxyhexosyl-glucuronide, was the main compound detected $(4.78 \mathrm{mg} / \mathrm{g}$, Table 4). Several biological properties, including antioxidant and antiproliferative activities, of different kaempferol glycosides has been previously reported (Calderón-Montaño et al., 2011; Xiao et al., 2014). The second most abundant compound in this fraction was tetragalloyl-glucose (compound 27) The biological activity of this compound has been described (Sugimoto et al., 2009; Xiang et al., 2010; Zhang et al., 2009). FM3 showed to be constituted by phenolic acids, flavonoids and ellagitannin derivatives, this later group being the most abundant $(13.1 \mathrm{mg} / \mathrm{g}$, Table 4). Fraction FM4 consisted mainly of ellagitannin derivatives $(57.8 \mathrm{mg} / \mathrm{g}$, Table 4$)$, together with a small amount of flavonoids ( $6.06 \mathrm{mg} / \mathrm{g}$, Table 4). Compound 14 (geraniin isomer 1) was the most abundant in both fractions FM3 and FM4 (5.1 and $38.7 \mathrm{mg} / \mathrm{g}$, respectively, Table 4).

Fractions FM7, FM8, FM11 and FM12 displayed high antioxidant activity but no relevant cytotoxic activity. With the exception of FM7, for which phenolic acids were the major constituents, in all the other fractions ellagitannin derivatives were the most abundant compounds. The major compound found in FM7 was tentatively identified as 3-Ocaffeoylquiic acid (compound 1), while for the remaining fractions geraniin isomer 1 (compound 14) was the most abundant compound (Table 4).

The EtOAc extract yielded, after fractionation, eleven fractions (FEA1-11). However, the color of fractions FEA1-FEA4 did not allow the study of their bioactivity, most probably due to the presence of chlorophylls or other pigments. The remaining fractions of this extract possessed antioxidant activity much lower than that of the fractions resulting from the acetone and $\mathrm{MeOH}$ extracts. Fractions FEA5, FEA6 and FEA8 showed moderate cytotoxicity. Fraction FEA5 showed to 
Table 5

Bioactive properties of different fractions of ethyl acetate, acetone and methanol extracts of Geranium robertianum $\mathrm{L}$.

\begin{tabular}{|c|c|c|c|c|c|c|c|c|}
\hline \multirow[b]{2}{*}{ Fractions } & \multicolumn{3}{|c|}{ Antioxidant activity $\left(\mathrm{EC}_{50}, \mu \mathrm{g} / \mathrm{mL}\right)$} & \multicolumn{4}{|c|}{ Antiproliferative activity $\left(\mathrm{GI}_{50}, \mu \mathrm{g} / \mathrm{mL}\right)$} & \multirow{2}{*}{$\begin{array}{l}\text { Hepatotoxicity }\left(\mathrm{GI}_{50}, \mu \mathrm{g} / \mathrm{mL}\right) \\
\text { PLP2 }\end{array}$} \\
\hline & DPPH & Reducing power & $\beta$-Carotene/linoleic acid & MCF-7 & NCI-H460 & HeLa & HepG2 & \\
\hline FEA1 & nd & nd & nd & nd & nd & nd & nd & nd \\
\hline FEA2 & nd & nd & nd & nd & nd & nd & nd & nd \\
\hline FEA3 & nd & nd & nd & nd & nd & nd & nd & nd \\
\hline FEA4 & nd & nd & nd & nd & nd & nd & nd & nd \\
\hline FEA5 & $119.0 \pm 0.3 \mathrm{e}$ & $974 \pm 2 c$ & $945 \pm 61 c$ & $92 \pm 1 d$ & $51 \pm 4 \mathrm{e}$ & $96 \pm 7 d$ & $92 \pm 10 \mathrm{e}$ & $128 \pm 5 c$ \\
\hline FEA6 & $302 \pm 5 d$ & $232 \pm 6 e$ & $448 \pm 43 c$ & $54 \pm 2 f$ & $119 \pm 2 d$ & $34 \pm 3 e$ & $72 \pm 3 e$ & $238 \pm 20 b$ \\
\hline FEA7 & $614 \pm 3 c$ & $396 \pm 2 d$ & $509 \pm 43 c$ & $90 \pm 5 d$ & $243 \pm 17 b$ & $89 \pm 2 d$ & $237 \pm 26 a$ & $>400$ \\
\hline FEA8 & $2777 \pm 35 a$ & $3617 \pm 77 a$ & $1628 \pm 146 b$ & $82 \pm 6 e$ & $169 \pm 6 c$ & $88 \pm 2 d$ & $83 \pm 5 e$ & $235 \pm 9 b$ \\
\hline FEA9 & $1516 \pm 128 b$ & $1591 \pm 6 b$ & $15128 \pm 943 a$ & $110 \pm 7 c$ & $232 \pm 19 b$ & $115 \pm 8 c$ & $125 \pm 15 d$ & $302 \pm 20 \mathrm{a}$ \\
\hline FEA10 & $260 \pm 12 d$ & $179 \pm 8 f$ & $529 \pm 59 c$ & $188 \pm 1 b$ & $236 \pm 23 b$ & $186.4 \pm 0.4 b$ & $153 \pm 5 c$ & $>400$ \\
\hline FEA11 & $271 \pm 7 d$ & $171 \pm 9 f$ & $610 \pm 16 c$ & $234 \pm 2 a$ & $304 \pm 18 \mathrm{a}$ & $236 \pm 6 a$ & $207 \pm 11 b$ & $>400$ \\
\hline \multicolumn{9}{|l|}{$p$-values $(\mathrm{n}=21)$} \\
\hline Homoscedasticity $^{1}$ & $<0.001$ & $<0.001$ & $<0.001$ & 0.246 & 0.337 & 0.256 & 0.252 & 0.071 \\
\hline 1-way ANOVA ${ }^{2}$ & $<0.001$ & $<0.001$ & $<0.001$ & $<0.001$ & $<0.001$ & $<0.001$ & $<0.001$ & $<0.001$ \\
\hline FA1 & $4062 \pm 75 b$ & $690 \pm 12 b$ & $1577 \pm 67 a$ & $87 \pm 4 \mathrm{~cd}$ & $240 \pm 15 a$ & $87 \pm 4 c$ & $93 \pm 9 \mathrm{~cd}$ & $286 \pm 15 a$ \\
\hline FA2 & $9903 \pm 18 a$ & $774 \pm 7 a$ & $1530 \pm 52 a$ & $195 \pm 22 b$ & $251 \pm 17 a$ & $223 \pm 12 a$ & $164 \pm 13 b$ & $>400$ \\
\hline FA3 & $1152 \pm 17 c$ & $648 \pm 9 c$ & $734 \pm 57 b$ & $91 \pm 8 c$ & $98 \pm 1 b c$ & $71 \pm 1 d$ & $251 \pm 26 a$ & $196 \pm 16 b$ \\
\hline FA4 & $52 \pm 3 g$ & $31.2 \pm 0.1 \mathrm{j}$ & $61 \pm 5 e$ & $237 \pm 7 a$ & $72 \pm 7 \mathrm{~cd}$ & $39 \pm 4 d$ & $56 \pm 3 e$ & $>400$ \\
\hline FA5 & $44 \pm 1 g$ & $32.1 \pm 0.2 \mathrm{j}$ & $67.4 \pm 0.2 \mathrm{e}$ & $48 \pm 9 d$ & $61.9 \pm 0.4 \mathrm{~d}$ & $67 \pm 3 d$ & $50 \pm 2 e$ & $127 \pm 12 \mathrm{e}$ \\
\hline FA6 & $57 \pm 2 g$ & $179 \pm 6 f$ & $226 \pm 9 c$ & $48 \pm 8 d$ & $231 \pm 16 a$ & $90 \pm 3 c$ & $74 \pm 4 \mathrm{de}$ & $172 \pm 18 b c d$ \\
\hline FA7 & $119 \pm 1 \mathrm{f}$ & $89 \pm 5 h$ & $125 \pm 7 d$ & $67 \pm 6 \mathrm{~cd}$ & $92 \pm 6 b c$ & $61 \pm 1 d$ & $60 \pm 4 e$ & $151 \pm$ 10de \\
\hline FA8 & $112 \pm 3 f$ & $46.9 \pm 0.3 \mathrm{i}$ & $35 \pm 1 \mathrm{e}$ & $62 \pm 3 \mathrm{~cd}$ & $117 \pm 2 b$ & $60 \pm 6 d$ & $63 \pm 2 e$ & $167 \pm 16 \mathrm{~cd}$ \\
\hline FA9 & $553 \pm 14 d$ & $269 \pm 9 e$ & $195 \pm 5 c$ & $191 \pm 18 b$ & $244 \pm 18 a$ & $171 \pm 10 b$ & $170 \pm 17 b$ & $>400$ \\
\hline FA10 & $439 \pm 37 e$ & $285 \pm 2 d$ & $239 \pm 12 c$ & $264 \pm 8 a$ & $247 \pm 20 a$ & $215 \pm 11 a$ & $267 \pm 23 a$ & $>400$ \\
\hline FA11 & $139 \pm 2 f$ & $108 \pm 2 g$ & $155 \pm 1 d$ & $77 \pm 7 \mathrm{~cd}$ & $259 \pm 21 a$ & $102 \pm 12 c$ & $101 \pm 4 c$ & $191 \pm 12 b c$ \\
\hline \multicolumn{9}{|l|}{$p$-values $(\mathrm{n}=33)$} \\
\hline Homoscedasticity ${ }^{1}$ & $<0.001$ & $<0.001$ & $<0.001$ & 0.089 & 0.242 & 0.236 & 0.110 & 0.072 \\
\hline 1 -way ANOVA ${ }^{2}$ & $<0.001$ & $<0.001$ & $<0.001$ & $<0.001$ & $<0.001$ & $<0.001$ & $<0.001$ & $<0.001$ \\
\hline FM1 & $13471 \pm 916 a$ & $1595 \pm 16 a$ & $1590 \pm 53 a$ & $95 \pm 6 c$ & $269 \pm 16 d$ & $138 \pm 13 e$ & $116 \pm 11 d$ & $322 \pm 33 b$ \\
\hline FM2 & $63 \pm 2 c$ & $147 \pm 8 \mathrm{e}$ & $913 \pm 65 b$ & $>400$ & $59 \pm 4 g$ & $53 \pm 6 g$ & $52 \pm 4 \mathrm{e}$ & $>400$ \\
\hline FM3 & $23 \pm 1 c$ & $51.5 \pm 0.1 \mathrm{ij}$ & $23 \pm 1 \mathrm{hi}$ & $74 \pm 1 c$ & $170 \pm 2 \mathrm{e}$ & $77 \pm 1 \mathrm{f}$ & $67 \pm 7 e$ & $207 \pm 10 c$ \\
\hline FM4 & $17.2 \pm 0.3 c$ & $44.6 \pm 0.7 j$ & $7.0 \pm 0.5 i$ & $78 \pm 1 c$ & $123 \pm 8 f$ & $78 \pm 1 f$ & $65 \pm 6 e$ & $166 \pm 13 d$ \\
\hline FM5 & $53 \pm 1 c$ & $95 \pm 1 \mathrm{f}$ & $207 \pm 11 \mathrm{e}$ & $247 \pm 9 a$ & $323 \pm 27 a b$ & $258 \pm 1 \mathrm{ab}$ & $215 \pm 8 c$ & $>400$ \\
\hline FM6 & $98 \pm 6 c$ & $188 \pm 3 c$ & $281 \pm 12 d$ & $213 \pm 12 \mathrm{ab}$ & $311 \pm 23 b c$ & $233 \pm 4 c$ & $241 \pm 12 b$ & $>400$ \\
\hline FM7 & $26 \pm 2 c$ & $63 \pm 8 g h$ & $101 \pm 10 f$ & $189 \pm 24 b$ & $279 \pm 18 \mathrm{~cd}$ & $203 \pm 20 d$ & $103 \pm 8 d$ & $>400$ \\
\hline FM8 & $49 \pm 1 c$ & $65.7 \pm 0.4 \mathrm{~g}$ & $85 \pm 1 \mathrm{fg}$ & $188 \pm 30 \mathrm{~b}$ & $353 \pm 11 a$ & $241 \pm 5 b c$ & $205 \pm 9 c$ & $>400$ \\
\hline FM9 & $82 \pm 1 c$ & $171 \pm 3 d$ & $89 \pm 9 f g$ & $223 \pm 17 \mathrm{ab}$ & $>400$ & $270 \pm 3 a$ & $259 \pm 15 b$ & $>400$ \\
\hline FM10 & $132 \pm 1 c$ & $232 \pm 4 b$ & $509 \pm 4 c$ & $250 \pm 1 \mathrm{a}$ & $332 \pm 22 \mathrm{ab}$ & $265 \pm 15 a$ & $252 \pm 14 b$ & $>400$ \\
\hline FM11 & $26.0 \pm 0.5 c$ & $33 \pm 1 \mathrm{k}$ & $69 \pm 4 f g$ & $222 \pm 12 \mathrm{ab}$ & $264 \pm 7 d$ & $255 \pm 18 \mathrm{ab}$ & $103 \pm 5 d$ & $>400$ \\
\hline FM12 & $36.5 \pm 0.4 c$ & $51 \pm 1 \mathrm{ij}$ & $52.9 \pm 0.5 \mathrm{gh}$ & $197 \pm 9 b$ & $305 \pm 22 b c$ & $204 \pm 10 d$ & $104 \pm 12 d$ & $367 \pm 27 a$ \\
\hline FM13 & $569 \pm 9 b$ & $56 \pm 1 \mathrm{hi}$ & $213 \pm 13 e$ & $178 \pm 15 b$ & $353 \pm 21 a$ & $200 \pm 5 d$ & $293 \pm 16 a$ & $>400$ \\
\hline \multicolumn{9}{|l|}{$p$-values $(\mathrm{n}=39)$} \\
\hline Homoscedasticity $^{1}$ & $<0.001$ & $<0.001$ & $<0.001$ & 0.016 & 0.324 & 0.112 & 0.874 & 0.006 \\
\hline 1-way ANOVA ${ }^{2}$ & $<0.001$ & $<0.001$ & $<0.001$ & $<0.001$ & $<0.001$ & $<0.001$ & $<0.001$ & $<0.001$ \\
\hline
\end{tabular}

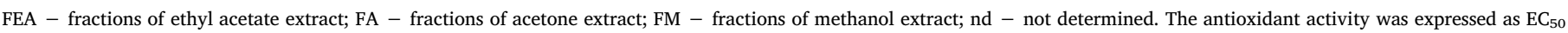

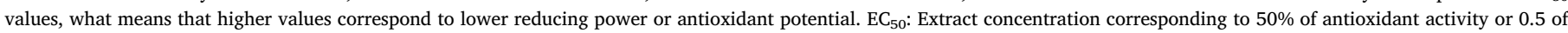

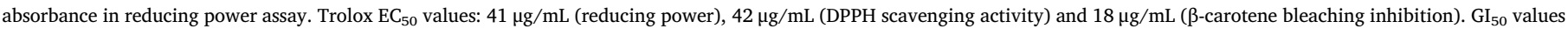

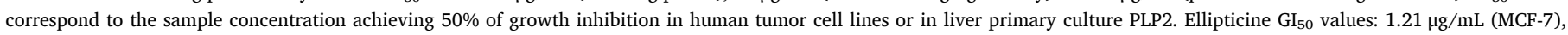
$1.03 \mu \mathrm{g} / \mathrm{mL}$ (NCI-H460), $0.91 \mu \mathrm{g} / \mathrm{mL}$ (HeLa), $1.10 \mu \mathrm{g} / \mathrm{mL}$ (HepG2) and $2.29 \mu \mathrm{g} / \mathrm{mL}$ (PLP2).

${ }^{1}$ Homoscedasticity among fractions of each exctract were tested by the Levene test: homoscedasticity, $p>0.05$; heteroscedasticity, $p<0.05$.

${ }^{2} p<0.05$ indicates that the mean value of at least one component differs from the others (in this case, multiple comparison tests were performed). For each fraction, means within a column with different letters differ significantly $(p<0.05)$.

contain only phenolic acids and, at the same time, the higher amount of this phenolic group among all the tested fractions. The major compound found in this fraction was $p$-hydroxybenzoic acid (compound 11, Table 2). Fractions FEA6 and FEA8 showed to contain, apart from phenolic acids, flavonoids as the main phenolic compounds. In both fractions, the most abundant compounds were derivatives of quercetin and kaempferol (Table 2).

The bioactivity of several fractions was found to be higher, in some cases considerably, than that of the corresponding extract. However, for the majority of fractions the bioactivity was lower what seems to point to a synergistic effect of the mixture of compounds present in the extract. In a number of fractions the hepatotoxicity displayed $\mathrm{GI}_{50}$ values higher than $400 \mathrm{mg} / \mathrm{mL}$, while for the whole extracts $\mathrm{GI}_{50}$ values ranged from 176 to $290 \mathrm{mg} / \mathrm{mL}$.

\subsection{Correlation between the phenolic profile and the bioactivity of the fractions}

The relationship between the sample's cytotoxic and antioxidant properties and the phenolic composition was established based on the correlation factors between total phenolic acids, total ellagitannin derivatives, total flavonoids and total phenolic compounds, and the $\mathrm{EC}_{50}$ and $\mathrm{GI}_{50}$ values found, respectively, for the antioxidant and cytotoxic activities (Table 6). In what concerns the fractions obtained from the EtOAc extract, there seems to exist no relevant correlation between phenolic composition and antioxidant activity. However, the cytotoxic activity of these fractions was correlated significantly with total flavonoids $\left(r^{2}=-0.814,-0.834\right.$ and -0.739 , for the NCI-H460, HepG2 and PLP2 cell lines, respectively). The total phenolic compounds of 
Correlation coefficients of phenolic compounds with bioactivity of the fractions of Geranium robertianum L. extracts.

\begin{tabular}{|c|c|c|c|c|c|c|c|c|c|c|c|c|c|}
\hline & & \multicolumn{4}{|c|}{ Fractions of EtOAc extract } & \multicolumn{4}{|c|}{ Fractions of Acetone extract } & \multicolumn{4}{|c|}{ Fractions of $\mathrm{MeOH}$ extract } \\
\hline & & TPA & TED & $\mathrm{TF}$ & TPC & TPA & TED & $\mathrm{TF}$ & TPC & TPA & TED & $\mathrm{TF}$ & TPC \\
\hline \multicolumn{14}{|l|}{ Antioxidant activity } \\
\hline \multirow[t]{2}{*}{ DPPH } & $r^{2}$ & -0.397 & - & 0.397 & 0.257 & -0.383 & -0.778 & - & -0.71 & -0.299 & -0.278 & 0.141 & -0.34 \\
\hline & $p$-values & 0.143 & - & 0.202 & 0.303 & 0.159 & $<0.001$ & - & $<0.001$ & 0.156 & 0.161 & 0.576 & 0.043 \\
\hline \multirow[t]{2}{*}{ Reducing Power } & $r^{2}$ & -0.091 & - & 0.466 & 0.437 & -0.784 & -0.672 & - & -0.842 & -0.215 & -0.334 & 0.225 & -0.583 \\
\hline & $p$-values & 0.748 & - & 0.127 & 0.07 & 0.001 & 0.002 & - & $<0.001$ & 0.312 & 0.089 & 0.37 & $<0.001$ \\
\hline \multirow[t]{2}{*}{$\beta$-Carotene/ linoleic acid } & $r^{2}$ & 0.112 & - & -0.652 & 0.375 & -0.959 & -0.545 & - & -0.801 & -0.159 & -0.324 & 0.693 & -0.264 \\
\hline & $p$-values & 0.69 & - & 0.022 & 0.125 & $<0.001$ & 0.019 & - & $<0.001$ & 0.459 & 0.099 & 0.001 & 0.12 \\
\hline \multicolumn{14}{|l|}{ Cytotoxic activity } \\
\hline \multirow[t]{2}{*}{ NCI-H460 } & $r^{2}$ & -0.686 & - & -0.814 & -0.514 & -0.704 & -0.645 & - & -0.853 & 0.075 & -0.376 & -0.652 & -0.576 \\
\hline & $p$-values & 0.005 & - & 0.001 & 0.029 & 0.003 & 0.004 & - & $<0.001$ & 0.727 & 0.053 & 0.003 & $<0.001$ \\
\hline \multirow[t]{2}{*}{ HeLa } & $r^{2}$ & 0.21 & - & -0.648 & 0.032 & -0.585 & -0.556 & - & -0.755 & 0.066 & -0.366 & -0.497 & -0.563 \\
\hline & $p$-values & 0.453 & - & 0.023 & 0.899 & 0.022 & 0.016 & - & $<0.001$ & 0.76 & 0.06 & 0.036 & $<0.001$ \\
\hline \multirow[t]{2}{*}{ HepG2 } & $r^{2}$ & -0.286 & - & -0.834 & -0.863 & -0.501 & -0.616 & - & -0.698 & -0.135 & -0.419 & -0.28 & -0.699 \\
\hline & $p$-values & 0.301 & - & 0.001 & $<0.001$ & 0.057 & 0.007 & - & $<0.001$ & 0.529 & 0.03 & 0.26 & $<0.001$ \\
\hline \multirow[t]{2}{*}{ MCF-7 } & $r^{2}$ & 0.151 & - & -0.658 & -0.011 & -0.439 & 0.491 & - & -0.192 & -0.094 & -0.454 & 0.552 & -0.362 \\
\hline & $p$-values & 0.592 & - & 0.02 & 0.966 & 0.101 & 0.039 & - & 0.37 & 0.663 & 0.017 & 0.018 & 0.03 \\
\hline \multirow[t]{2}{*}{ PLP2 } & & -0.621 & - & -0.739 & -0.698 & -0.473 & 0.435 & - & -0.258 & 0.24 & -0.7 & -0.031 & -0.613 \\
\hline & $p$-values & 0.013 & - & 0.006 & 0.001 & 0.075 & 0.071 & - & 0.224 & 0.259 & $<0.001$ & 0.903 & $<0.001$ \\
\hline
\end{tabular}

TPA - Total phenolic acids. TED - Total ellagitannins derivate. TF - Total flavonoids. TPC - Total phenolic compounds. $r^{2}-$ Correlation coefficient.

these fractions also showed significant correlation $\left(r^{2}=-0.863\right)$ with the toxicity against the HepG2 cell line.

Acetone fractions showed the highest correlation between total phenolic acids and the antioxidant activity determined by the $\beta$-carotene/linoleic acid assay $\left(r^{2}=-0.959\right)$, and a significant correlation $\left(\mathrm{r}^{2}=-0.784\right)$ between the total phenolic acids and the antioxidant activity assessed by the reducing power assay. The total ellagitannin derivatives showed good correlation with the results of the DPPH assay $\left(r^{2}=-0.778\right)$. The total phenolic compounds and the antioxidant activity of the acetone fractions showed to be significantly correlated $\left(r^{2}=-0.710,-0.842\right.$ and -0.801 , for DPPH, reducing power and $\beta$ carotene/linoleic acid assays, respectively). The cytotoxic effects of these fractions and the respective phenolic composition also presented relatively good correlation factors, being the cytotoxicity displayed against the NCI-H460 cell line correlated with total phenolic acids $\left(\mathrm{r}^{2}=-0.704\right)$ and the cytotoxicity observed against the NCI-H460, HeLa and HepG2 cell lines correlated with total phenolic compounds $\left(r^{2}=-0.853,-0.755\right.$ and -0.698 , respectively). The activities of the fractions obtained from the $\mathrm{MeOH}$ extract revealed to be poorly correlated with the phenolic composition. The best correlation was found between total phenolic compounds and the cytotoxicity observed on the HepG2 cell line $\left(r^{2}=-0.69\right)$.

\section{Conclusions}

Following a recent work conducted by our research group in which the bioactivity of different organic extracts of $G$. robertianum was studied, in this work the most bioactive extracts (EtOAC, acetone and $\mathrm{MeOH}$ ) were fractionated by gradient elution column chromatography. The phenolic profile of the resulting fractions was determinated and the antioxidant and cytotoxic activities were evaluated and correlated with the respective phenolic composition. In addition to hydrolyzable tannins, the most abundant compounds found in this plant, were also identified flavonoid glycosides derivatives - mainly kaempferol and quercetin derivatives - and phenolic acids. To the best of our knowledge, about two dozen of the compounds found were identified in $G$. robertianum for the first time. In general, the fractions from the acetone extract were those exhibiting higher bioactivity when compared with those from the other extracts. Three fractions of acetone extract (FA4, FA5 and FA8) displayed consistently low $\mathrm{EC}_{50}$ and $\mathrm{GI}_{50}$ values. These fractions also presented the higher contents of total phenolic compounds when compared with the other fractions. Fraction FA4 displayed particularly low hepatotoxicity $\left(\mathrm{IC}_{50}>400 \mu \mathrm{g} / \mathrm{mL}\right)$. Generally, the fractions resulting from the $\mathrm{MeOH}$ extract possessed moderate biological activity. The EtOAc fractions presented antioxidant activity much lower than that of the fractions resulting from the acetone and $\mathrm{MeOH}$ extracts. Some of these fractions showed moderate cytotoxicity. The acetone fractions were the ones that presented the highest correlation factors between biological activity and phenolic composition.

\section{Acknowledgements}

This work was supported by European Investment Funds by FEDER/ COMPETE/POCI- Operational Competitiveness and Internationalization Programme, under Project POCI-01-0145-FEDER-006958 and National Funds by FCT - Portuguese Foundation for Science and Technology under the projects UID/AGR/04033, UID/AGR/00690/2013 (CIMO) and UID/QUI/00616/2013 (CQ-VR). V.C. Graça, M.I. Dias and L. Barros thank FCT for SFRH/BD/52542/2014, SFRH/BD/84485/2012, and SFRH/BPD/107855/2015 grants, respectively.

\section{References}

Al-Ayed, A.S., 2015. Integrated mass spectrometry approach to screening of phenolic molecules in Hyphaene thebiaca fruits with their antiradical activity by thin-layer chromatography. Indian J. Chem. Technol. 22, 155-161.

Allen, D.E., Hatfield, G., 2004. Medicinal Plants in Folk Tradition: An Ethnobotany of Britain \& Ireland. Timber Press, Portland, Cambridge.

Amaral, S., Mira, L., Nogueira, J.M.F., Silva, A.P., Florêncio, M.H., 2009. Plant extracts with anti-inflammatory properties? A new approach for characterization of their bioactive compounds and establishment of structure-antioxidant activity relationships. Bioorg. Med. Chem. 17, 1876-1883.

Aprioku, J.S., 2013. Pharmacology of free radicals and the impact of reactive oxygen species on the testis. J. Reprod. Infertil. 14, 158-172.

Barros, L., Pereira, E., Calhelha, R.C., Dueñas, M., Carvalho, A.M., Santos-Buelga, C., Ferreira, I.C.F.R., 2013. Bioactivity and chemical characterization in hydrophilic and lipophilic compounds of Chenopodium ambrosioides L. J. Funct. Foods 5, 1732-1740.

Bastos, D.H., Saldanha, L.A., Catharino, R.R., Sawaya, A., Cunha, I.B., Carvalho, P.O., Eberlin, M.N., 2007. Phenolic antioxidants identified by ESI-MS from yerba maté (Ilex paraguariensis) and green tea (Camelia sinensis) extracts. Molecules 12, 423-432.

Calderón-Montaño, J.M., Burgos-Morón, E., Pérez-Guerrero, C., López-Lázaro, M., 2011. A Review on the dietary flavonoid kaempferol. Mini Rev. Med. Chem. 11, 298-344.

Carocho, M., Ferreira, I.C.F.R., 2013. A review on antioxidants, prooxidants and related controversy Natural and synthetic compounds, screening and analysis methodologies and future perspectives. Food Chem. Toxicol. 51, 15-25.

Cheng, H.S., Ton, S.H., Kadir, K.A., 2017. Ellagitannin geraniin: a review of the natural sources, biosynthesis, pharmacokinetics and biological effects. Phytochem. Rev. 16, 159-193.

Clifford, M.N., Johnston, K.L., Knight, S., Kuhnert, N., 2003. Hierarchical scheme for LC- 
MSn identification of chlorogenic acids. J. Agric. Food Chem. 51, 2900-2911.

Clifford, M.N., Knight, S., Kuhnert, N., 2005. Discriminating between the six isomers of dicaffeoylquinic acid by LC-MSn. J. Agric. Food Chem. 53, 3821-3832.

Cunha, A.P., Silva, A.P., Roque, A.R., 2012. Plantas E Produtos Vegetais Em Fitoterapia, 4th ed. Fundação Calouste Gulbenkian, Lisboa.

Dias, M.I., Barros, L., Dueñas, M., Pereira, E., Carvalho, A.M., Alves, R.C., Oliveira, M.B.P.P., Santos-Buelga, C., Ferreira, I.C.F.R., 2013. Chemical composition of wild and commercial Achillea millefolium L. and bioactivity of the methanolic extract infusion and decoction. Food Chem. 141, 4152-4160.

Dias, M.I., Barros, L., Oliveira, M.B.P.P., Santos-Buelga, C., Ferreira, I.C.F.R., 2015 Phenolic profile and antioxidant properties of commercial and wild Fragaria vesca L. roots: a comparison between hydromethanolic and aqueous extracts. Ind. Crops Prod. 63, 125-132.

Fernandes, A., Fernandes, I., Cruz, L., Mateus, N., Cabral, M., Freitas, V., 2009. Antioxidant and biological properties of bioactive phenolic compounds from Quercus suber L. J. Agric. Food Chem. 57, 11154-11160.

Fodorea, C.S., Vlase, L., Suciu, S., Tamas, M., Leucuta, S.E., 2005. Preliminary HPLC study on some polyphenols of Geranium robertianum L. (Geraniaceae). Rev. Med. Chir. Soc. Med. Nat. Iasi 109, 174-178.

Fridrich, D., Glabasnia, A., Fritz, J., Esselen, M., Pahlke, G., Hofmann, T., Marko, D., 2008. Oak ellagitannins suppress the phosphorylation of the epidermal growth factor receptor in human colon carcinoma cells. J. Agric. Food Chem. 56, 3010-3015.

Galleano, M., Verstraeten, S.V., Oteiza, P.I., Fraga, C.G., 2010. Antioxidant actions of flavonoids: thermodynamic and kinetic analysis. Arch. Biochem. Biophys. 501, 23-30.

Graça, V.C., Ferreira, I.C.F.R., Santos, P.F., 2016a. Phytochemical composition and biological activities of Geranium robertianum L.: a review. Ind. Crops Prod. 87, 363-378.

Graça, V.C., Barros, L., Calhelha, R.C., Dias, M.I., Carvalho, A.M., Santos-Buelga, C., Santos, P.F., Ferreira, I.C.F.R., 2016b. Chemical characterization and bioactive properties of aqueous and organic extracts of Geranium robertianum L. Food Funct. 7, 3807-3814.

Hanhineva, K., Rogachev, I., Kokko, H., Mintz-Oron, S., Venger, I., Kärenlampi, S., Aharoni, A., 2008. Non-targeted analysis of spatial metabolite composition in strawberry (Fragaria x ananassa) flowers. Phytochemistry 69 2463-2248.

Harborne, J.B., Williams, C.A., 2002. Phytochemistry of the genus Geranium. In: LisBalchin, M. (Ed.), Geranium and Pelargonium - The Genera Geranium and Pelargonium. Taylor \& Francis, London, pp. 20-29.

Ivancheva, S., Petrova, A., 2000. A chemosystematic study of eleven Geranium species. Biochem. Syst. Ecol. 28, 255-260.

Jordão, A.M., Correia, A.C., DelCampo, R., SanJosé, M.L.G., 2012. Antioxidant capacity, scavenger activity, and ellagitannins content from commercial oak pieces used in winemaking. Eur. Food Res. Technol. 235, 817-825.

Kartnig, T., Bucar-Stachel, J., 1991. Flavonoide aus den oberirdischen Teilen von Geranium robertianum (Flavonoids from the aerial parts of Geranium robertianum). Planta Med. 57, 292-293.

Kobakhidze, K.B., Alaniya, M.D., 2004. Flavonoids from Geranium robertianum. Chem. Nat. Compd. 40, 89-90.

Neagu, E., Paun, G., Constantin, D., Radu, G.L., 2013. Cytostatic activity of Geranium robertianum L. extracts processed by membrane procedures. Arab. J. Chem. http:// dx.doi.org/10.1016/j.arabjc.2013.09.028.
Okuda, T., Mori, K., Seno, K., Hatano, T., 1979. Constituents of Geranium thunbergii sieb. et zucc. VII. High-performance reversed-phase liquid chromatography of hydrolysable tannins and related polyphenols. J. Chromatogr. 171, 313-320.

Okuda, T., Mori, K., Hatano, T., 1980. The distribution of geraniin and mallotusinic acid in the order geraniales. Phytochemistry 19, 547-551.

Okuda, T., Yoshida, T., Hatano, T., 2000. Correlation of oxidative transformations of hydrolyzable tannins and plant evolution. Phytochemistry 55, 513-529.

Paun, G., Neagu, E., Tache, A., Radu, G.L., Parvulescu, V., 2011. Application of the nanofiltration process for concentration of polyphenolic compounds from Geranium robertianum and Salvia officinalis extracts. Chem. Biochem. Eng. Q 25, 453-460.

Paun, G., Neagu, E., Litescu, S.C., Rotinberg, P., Radu, G.L., 2012. Application of membrane processes for the concentration of Symphytum officinale and Geranium robertianum extracts to obtain compounds with high anti-oxidative activity. J. Serb. Chem. Soc. 77, 1191-1203.

Paun, G., Litescu, S.C., Neagu, E., Tache, A., Radu, G.L., 2014. Evaluation of Geranium spp., Helleborus spp. and Hyssopus spp. polyphenolic extracts inhibitory activity against urease and $\alpha$-chymotrypsin. J. Enzyme Inhib. Med. Chem. 29, 28-34.

Piwowarski, J.P., Granica, S., Zwierzyńska, M., Stefańska, J., Schopohl, P., Melzig, M.F., Kiss, A.K., 2014. Role of human gut microbiota metabolism in the anti-inflammatory effect of traditionally used ellagitannin-rich plant materials. J. Ethnopharmacol. 155, 801-809.

Roriz, C.L., Barros, L., Carvalho, A.M., Santos-Buelga, C., Ferreira, I.C.F.R., 2014. Pterospartum tridentatum, Gomphrena globosa and Cymbopogon citratus: a phytochemical study focused on antioxidant compounds. Food Res. Int. 62, 684-693.

Rubió, L., Motilva, M.-J., Romero, M.-P., 2013. Recent advances in biologically active compounds in herbs and spices A review of the most effective antioxidant and antiInflammatory active principles. Crit. Rev. Food Sci. Nutr. 53, 943-953.

Sugimoto, K., Nakagawa, K., Hayashi, S., Amakura, Y., Yoshimura, M., Yoshida, T., Yamaji, R., Nakano, Y., Inui, H., 2009. Hydrolyzable tannins as antioxidants in the leaf extract of Eucalyptus globulus possessing tyrosinase and hyaluronidase inhibitory activities. Food Sci. Technol. Res. 15, 331-336.

Tuominen, A., Toivonen, E., Mutikainen, P., Salminen, J.-P., 2013. Defensive strategies in Geranium sylvaticum. Part 1: Organ-specific distribution of water-soluble tannins, flavonoids and phenolic acids. Phytochemistry 95, 394-407.

Tuominen, A., 2013. Defensive strategies in Geranium sylvaticum, Part 2: Roles of watersoluble tannins, flavonoids and phenolic acids against natural enemies. Phytochemistry 95, 408-420.

Wu, Q.-Y., Zhou, Y., Jin, X., Guan, Y., Xu, M., Liu, L.-F., 2011. Chromatographic fingerprint and the simultaneous determination of five bioactive components of Geranium carolinianum L. Water extract by high performance liquid chromatography. Int. J. Mol. Sci. 12, 8740-8749.

Xiang, Y-f., Ju, H.-Q., Li, S., Zhang, Y.-J., Yang, C.-R., Wang, Y.-F., 2010. Effects of 1,2,4,6-tetra-O-galloyl- $\beta$-D-glucose from P. emblica on HBsAg and HBeAg secretion in HepG2.2.15 cell culture. Virol. Sin. 25, 375-380.

Xiao, J., Chen, T., Cao, H., 2014. Flavonoid glycosylation and biological benefits. Biotechnol. Adv. http://dx.doi.org/10.1016/j.biotechadv.2014.05.004.

Zhang, J., Li, L., Kim, S.-H., Hagerman, A.E., Lü, J., 2009. Anti-cancer, anti-diabetic and other pharmacologic and biological activities of penta-galloyl-glucose. Pharm. Res. 26, 2066-2080. 